\title{
Study of Dielectric Relaxation Behavior of Liquid Crystal Copolyester Vectra-A by Thermally Stimulated Discharge Current Technique
}

\author{
Sapna Kalia, J. K. Sharma, and Vandana Sharma \\ Department of Physics, Maharishi Markandeshwar University Mullana, Ambala, Haryana 133 207, India \\ Correspondence should be addressed to Sapna Kalia; skalia19@gmail.com
}

Received 31 January 2013; Accepted 27 February 2013

Academic Editors: A. Hashidzume and A. Mousa

Copyright (C) 2013 Sapna Kalia et al. This is an open access article distributed under the Creative Commons Attribution License, which permits unrestricted use, distribution, and reproduction in any medium, provided the original work is properly cited.

\begin{abstract}
The dielectric relaxation behavior of thermotropic liquid crystal copolyester of $73 \%$ of p-hydroxy-benzoic acid (HBA) and $27 \%$ of 2-hydroxy-6-naphthoic acid (HNA) (Vectra-A) at poling temperature $80^{\circ} \mathrm{C}$ has been studied using thermally stimulated depolarization current (TSDC) technique in the temperature range from $15^{\circ} \mathrm{C}$ to $250^{\circ} \mathrm{C}$. The TSD currents were obtained for different polarizing fields ranging from $3.8 \mathrm{kV} / \mathrm{cm}$ to $19.2 \mathrm{kV} / \mathrm{cm}$. TSD current spectra in the temperature range from $15^{\circ} \mathrm{C}$ to $250^{\circ} \mathrm{C}$ show three current maxima around $25^{\circ} \mathrm{C}, 110^{\circ} \mathrm{C}$, and $220^{\circ} \mathrm{C}$. The maxima around $25^{\circ} \mathrm{C}$ and $110^{\circ} \mathrm{C}$ correspond to characteristic dipolar relaxations $\beta$ and $\alpha$, respectively. The peak around $220^{\circ} \mathrm{C}$ is due to space charge effects named as $\delta$-relaxation. The various relaxation parameters like activation energy $(U)$, relaxation strength $(\Delta \varepsilon)$, preexponential factor $\left(\tau_{0}\right)$, the quantity of charge released $(Q)$ and concentration of trap $\left(N_{t}\right)$ for $\beta$ - and $\alpha$-relaxations at polarizing temperature $80^{\circ} \mathrm{C}$ for different polarizing fields were evaluated using Bucci-Fieschi fit. The linear variation between activation energy and natural logarithm of preexponential factor indicates the presence of compensation effect for dipolar relaxations of Vectra-A under present poling conditions.
\end{abstract}

\section{Introduction}

Liquid crystal copolymers of hydroxybenzoic and hydroxynaphthoic acids have received much interest due to their high chemical resistant which makes them suitable for making surgical instruments; their easily molded sheets have very good dimensional accuracy and strength and stiffness, and high strength-to-weight ratio makes them suitable for metal replacement applications. The dielectric and electrical properties of liquid crystal polymer (LCP) are strongly influenced by the molecular order and orientation. Thermally stimulated discharge current (TSDC) is a powerful tool for determining the characteristics of dielectric relaxation behavior of polymeric materials [1-4]. The advantage of this technique is its high sensitivity. In the present work, TSDC technique has been used to investigate the dielectric relaxation behavior of liquid crystal copolyester of $73 \%$ of p-hydroxybenzoic acid (HBA) and 27\% of 2-hydroxy-6-napthoic acid (HNA) commercially known as Vectra-A in the temperature range from $15^{\circ} \mathrm{C}$ to $250^{\circ} \mathrm{C}$ at poling temperature $80^{\circ} \mathrm{C}$. The TSD currents were obtained as a function of temperature for polarizing fields ranging from $3.8 \mathrm{kV} / \mathrm{cm}$ to $19.2 \mathrm{kV} / \mathrm{cm}$. The TSDC spectra were analyzed using Bucci-Fieschi-Guidi analysis [5]. The relaxation parameters activation energy, relaxation strength, preexponential factor, charge released, and concentration of trap density were determined from BFG plots. The effect of poling field on TSDC spectra and relaxation parameters is also discussed in the present paper. The linear variation of activation energy with natural logarithm of preexponential factor indicates the presence of compensation effect for dipolar relaxations of Vectra-A under present polarizing conditions.

\section{Theory}

The stored polarization's $P(t)$, decay rate $d P(t) / d t$, and dipolar relaxation time $\tau(T)$ are expressed by

$$
\frac{d P(t)}{d t}=-\frac{P(t)}{\tau(T)} .
$$


The uniform decay rate of dielectric is a function of time and temperature. The solution of (1) is

$$
P(t)=P_{0} \exp \left(-\int_{0}^{t} \frac{d t}{\tau(T)}\right)
$$

In case of freely rotating dipoles, the dielectric is polarized by application of an electric field $\left(E_{p}\right)$ and temperature $\left(T_{p}\right)$. Then,

$$
P_{0}=\frac{N \mu^{2} E_{p}}{3 k T_{p}}
$$

where $N$ is the concentration of dipoles, $k$ is Boltzmann's constant, and $\mu$ is the dipole moment.

The rate at which polarization changes or in other words depolarization current density is given by

$$
J(T)=-\frac{d P(t)}{d t}=\frac{P_{0}}{\tau} \exp \left(-\int_{0}^{t} \frac{d t}{\tau(T)}\right) .
$$

From (1) and (4), we obtain

$$
\begin{gathered}
P(t)=-\int_{0}^{t} J(T) d t, \\
J(T)=\frac{-P(t)}{\tau(T)} .
\end{gathered}
$$

Therefore,

$$
J(T)=\frac{\int_{0}^{t} J(T) d t}{\tau(T)} .
$$

In order to obtain the current density produced by the progressive decrease in polarization in the course of TSDC experiment where time and temperature are simultaneously varied, the differentiation must be done by taking $T$ as a new variable. The parameter $T$ is linearly increasing from a temperature $T_{0}$ according to the following relation:

$$
T=T_{0}+h t
$$

Here, $T_{0}$ is initial temperature and $h$ is the heating rate, that is, $h=d T / d t$.

By making the use of (6), we get

$$
\tau(T)=\frac{\int_{T}^{\infty} J(T) d T}{h J(T)} .
$$

Thus, the relaxation time $\tau(T)$ at any temperature can be calculated as follows:

$$
\tau(T)=\frac{\text { Area under the TSDC curve between } T \text { and } T_{\infty}}{\text { Value of TSD current at temperature } T} .
$$

The Arrhenius equation for the variation of $\tau$ with temperature is given as follows:

$$
\tau(T)=\tau_{0} \exp \left(\frac{U}{k T}\right),
$$

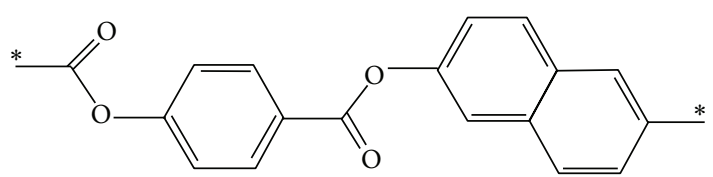

FIGURE 1: Structure of Vectra-A.

where $\tau_{0}$ is the natural relaxation time and $U$ is the activation energy needed to disorient a dipole. The energy $U$ can be seen as a potential barrier which the dipole has to surmount before it can readjust its direction

$$
\ln \tau(T)=\ln \tau_{0}+\frac{U}{k T}
$$

Thus, the graph between $\ln \tau(T)$ and $1 / T$ is a straight line (BFG plot) whose slope gives the value of activation energy $[5,6]$.

The quantity of charge released $(Q)$ is calculated from TSDC curve [7]. The concentration of the traps has been estimated using the following relation:

$$
N_{t}=\frac{Q}{(S L e G)}
$$

Here, $S$ and $L$ are the area and thickness of the sample, respectively, $e$ is the electronic charge, and $G$ is the photoconductivity gain, which is equal to the number of electrons passing through the sample for each absorbed photon. The value of $G$ is taken unity for the calculation of concentration of the traps [8].

\section{Material and Method}

The liquid crystal copolyester commercially known as Vectra$\mathrm{A}$ is a random copolyester of hydroxybenzoic acid (HBA) and hydroxynaphthoic acid (HNA), composed of $27 \%$ of HNA and $73 \%$ of HBA. Vectra-A consists of rigid rods like molecules that are ordered in the melt phase to form liquid crystal structures [9]. It has operating temperature up to $300^{\circ} \mathrm{C}$, very low-melt viscosity, excellent dimensional stability, very high tensile strength, and very high impact resistance [10]. The rigid rod-like macromolecules of VectraA are aligned in the melt state to produce its structure, as shown in Figure 1.

The Vectra-A, ivory in color, is procured in the form of rod having a diameter of 18 millimeters and a length of one meter from Goodfellow Cambridge limited Ermine Business Park, Huntingdon PE296WR, UK. The Samples were prepared by cutting the rod in the form of pellets of 1.3 millimeter thickness. The samples were metalized on both surfaces with aluminum by vacuum evaporation. The TSDC technique used to study the dielectric relaxation behavior of Vectra-A is shown in Figure 2.

The procedure for preparing thermoelectrets for the measurement of TSDC spectra is as follows.

(1) Samples of Vectra-A were heated up to $80^{\circ} \mathrm{C}$ in the specially designed temperature controlled furnace. 


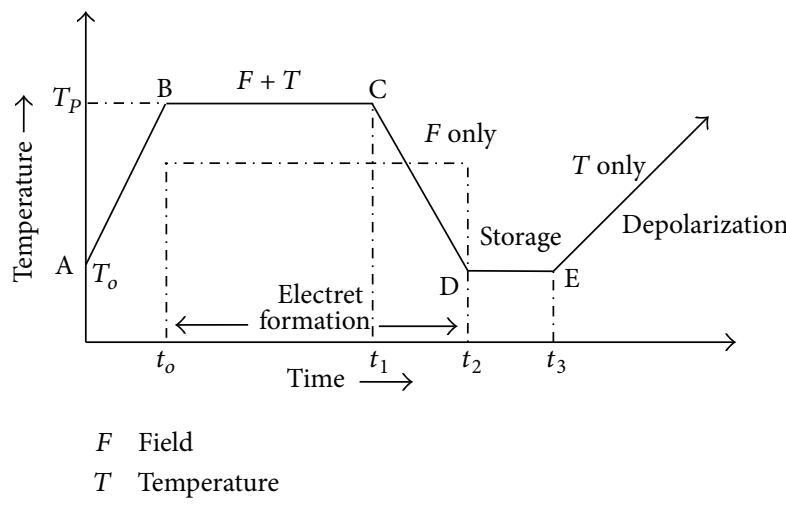

FIgUre 2: The TSDC technique.

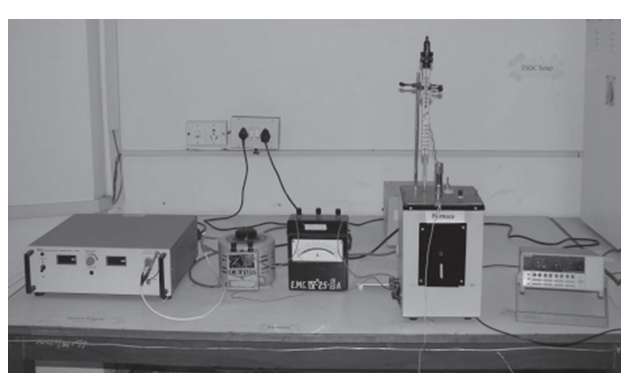

FIgURE 3: Experimental setup for TSDC.

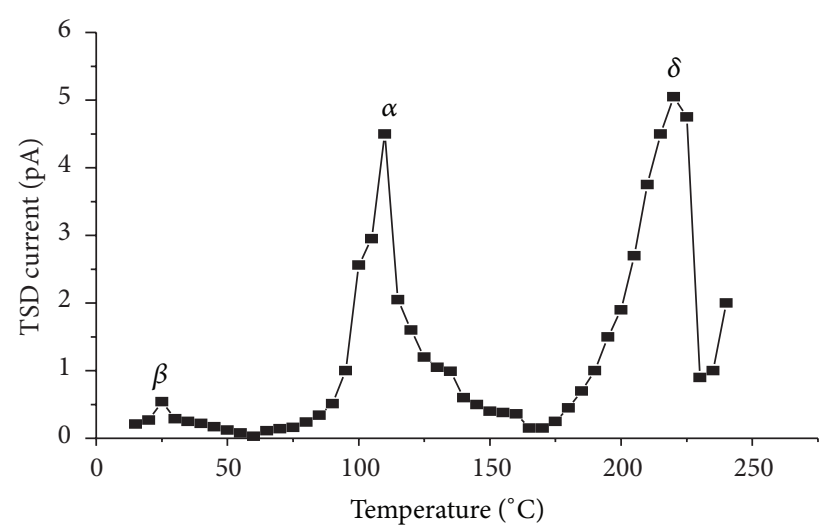

FIGURE 4: TSDC plot for polarizing field $7.7 \mathrm{kV} / \mathrm{cm}$ at polarizing temperature $T_{P}=80^{\circ} \mathrm{C}$.

(2) The thermoelectrets at $T_{P}=80^{\circ} \mathrm{C}$ were prepared by subjecting them to dc fields $E_{P}$ (from $3.8 \mathrm{kV} / \mathrm{cm}$ to $19.2 \mathrm{kV} / \mathrm{cm}$ ).

(3) The polarizing voltage was supplied using high voltage Aplab power supply for 1 hour.

(4) The samples were cooled to room temperature in the presence of electric field.

After this, both of the electrodes were short circuited for 24 hours. The sample is then reheated at constant rate of $2^{\circ} \mathrm{C} /$ minute. The depolarization current was measured with sensitive Keithley Electrometer 6514 procured from Keithley

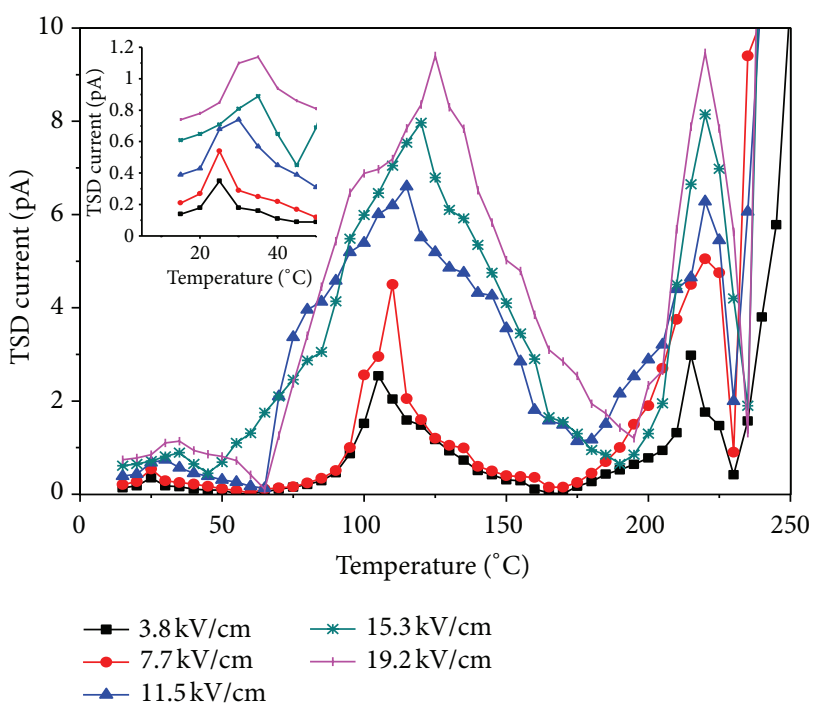

FIGURE 5: The TSDC spectra of Vectra-A samples at different electric fields $\left(T_{P}=80^{\circ} \mathrm{C}\right)$.

Instruments, Inc., Cleveland, OH, USA. The experimental setup for the present investigations is shown in Figure 3.

\section{Results and Discussion}

The TSD current spectra of Vectra-A at polarizing field $7.7 \mathrm{kV} / \mathrm{cm}$ in the temperature range from $15^{\circ} \mathrm{C}$ to $250^{\circ} \mathrm{C}$ is shown in Figure 4. The TSDC spectrum reveals three maxima, around $25^{\circ} \mathrm{C}, 110^{\circ} \mathrm{C}$, and $220^{\circ} \mathrm{C}$ which are termed as $\beta$-relaxation, $\alpha$-relaxation, and $\delta$-relaxation, respectively. The $\beta$-relaxation located at low temperature is related to the local motion of HNA groups as this motion is accompanied by reduced conformational constraints. Also, at low temperature due to steric hindrance, only naphthalene with their ester groups will rotate as a single unit [11]. The peak observed at high temperatures around $110^{\circ} \mathrm{C}$, namely, $\alpha$-relaxation is associated with the glass transition $[1,2]$. The glass transition is attributed to the cooperative rotation of main chain segments. Lukacs [12] also reported $\beta$ - and $\alpha$-relaxations around $25^{\circ} \mathrm{C}$ and $110^{\circ} \mathrm{C}$, respectively, by studying the temperaturedependent time resolve fluorescence spectra of Vectra-A. The $\delta$-relaxation is known as a space charge relaxation. The space charges are frozen at low temperatures, but with the increase in temperature, they gain their mobility and contribute to space charge relaxation.

In order to study the effect of polarizing field, the TSD spectra at different polarizing fields ranging from $3.8 \mathrm{kV} / \mathrm{cm}$ to $19.2 \mathrm{kV} / \mathrm{cm}$ are plotted as a function of temperature shown in Figure 5. From the figure, it is clear that there is significant effect of polarizing field on the magnitude as well as on the positions of $\beta$ - and $\alpha$-relaxations. The position of $\beta$ relaxation changes in the range from $25^{\circ} \mathrm{C}$ to $35^{\circ} \mathrm{C}$ and that of $\alpha$-relaxation in the range from $105^{\circ} \mathrm{C}-125^{\circ} \mathrm{C}$ with an increasing polarizing field in the range from $3.8 \mathrm{kV} / \mathrm{cm}$ to $19.2 \mathrm{kV} / \mathrm{cm}$. This shift with the increase in the polarizing field may be attributed to the fact that in liquid crystal 


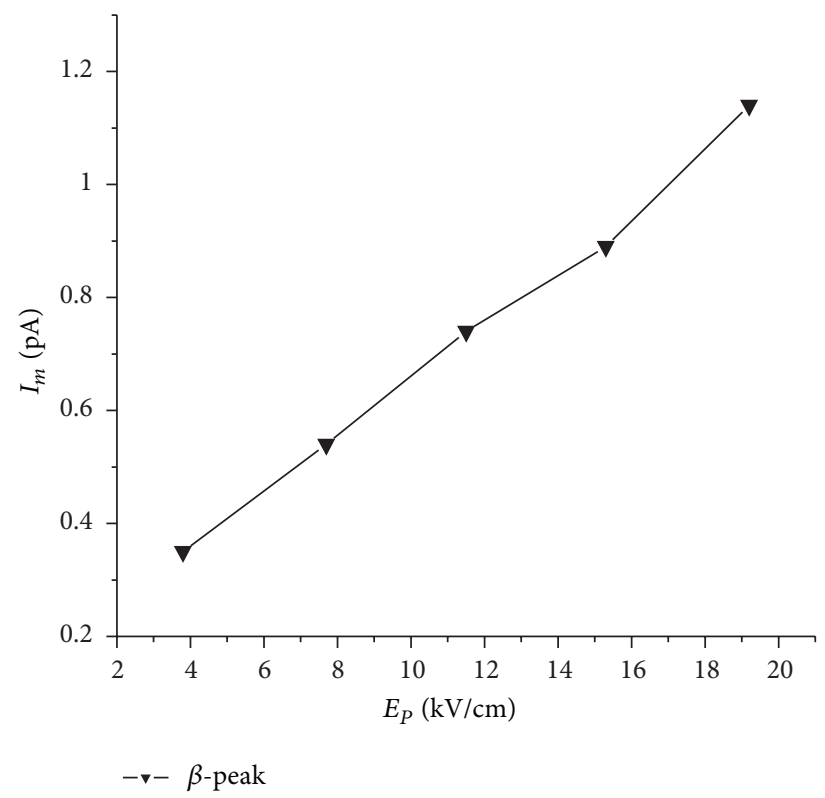

(a)

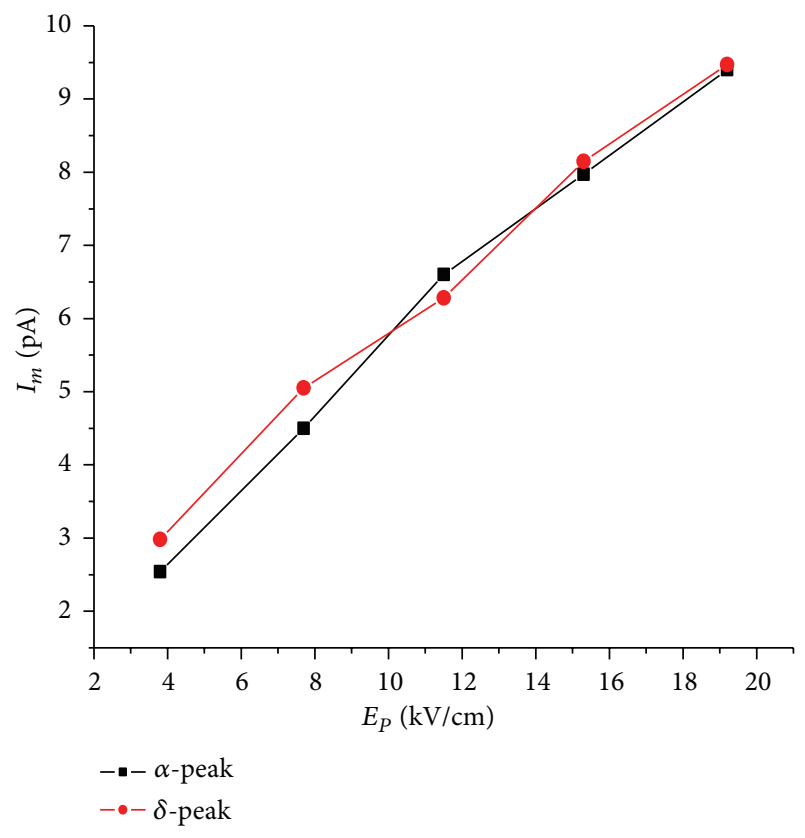

(b)

FIGURE 6: (a) Field dependence of maximum current $\left(I_{m}\right)$ for $\beta$-peak. (b) Field dependence of maximum current $\left(I_{m}\right)$ for $\alpha$ and $\delta$-peaks.

TABLE 1: Values of activation energy $(U)$, dipolar relaxation strength $\Delta \varepsilon$, preexponential factor $\left(\tau_{0}\right)$, charge released $(Q)$, and concentration of traps $\left(N_{t}\right)$ for $\beta$-relaxation at poling temperature $T_{P}=80^{\circ} \mathrm{C}$ for various polarizing fields.

\begin{tabular}{lccccc}
\hline \multicolumn{5}{c}{$\beta$-relaxation } \\
$\begin{array}{l}\text { Poling fields } \\
(\mathrm{kV} / \mathrm{cm})\end{array}$ & $U(\mathrm{eV})$ & $\Delta \varepsilon$ & $\tau_{0}(\mathrm{Sec})$ & $Q(\mathrm{C})$ & $N_{t}\left(\mathrm{~m}^{-3}\right)$ \\
\hline 3.8 & 0.51 & 0.27 & $1.74 \times 10^{-6}$ & $2.27 \times 10^{-10}$ & $6.19 \times 10^{11}$ \\
7.7 & 0.49 & 0.27 & $3.54 \times 10^{-6}$ & $4.65 \times 10^{-10}$ & $12.65 \times 10^{11}$ \\
11.5 & 0.42 & 0.32 & $6.83 \times 10^{-5}$ & $8.30 \times 10^{-10}$ & $22.59 \times 10^{11}$ \\
15.3 & 0.38 & 0.34 & $2.04 \times 10^{-4}$ & $11.56 \times 10^{-10}$ & $31.47 \times 10^{11}$ \\
19.2 & 0.32 & 0.34 & $3.39 \times 10^{-3}$ & $14.85 \times 10^{-10}$ & $40.42 \times 10^{11}$ \\
\hline
\end{tabular}

polymers, the length of spacer shrinks, and the results in increasing the rigidity of the molecules hence shift the peak to a higher temperature [13]. The position of $\delta$-relaxation is nearly independent of a polarizing field.

The magnitude of maximum current $\left(I_{m}\right)$ for $\beta-, \alpha-$, and $\delta$-relaxations are plotted with polarizing electric fields and shown in Figures 6(a) and 6(b). The plot shows that maximum current $\left(I_{m}\right)$ depends on the strength of polarizing field for a given poling temperature. The maximum current $\left(I_{m}\right)$ increases linearly with the polarizing fields. The linear increase of $I_{m}$ with the increase in the applied field for $\alpha$ and $\beta$-relaxations is confirming the presence of uniform bulk polarization and hence dipolar nature of these relaxations [14]. An increase in current maxima of $\delta$-peak, that is, located at higher temperature is attributed to the space charge effects that are the increasing with increasing polarizing field [1].
It is clear from Figure 5 that the glass transition of this aromatic copolyester is broadened, at higher polarizing fields. The broad glass transition in the liquid crystal polymers may be attributed to structural heterogeneities of the chains [15]. Various studies such as calorimetric [16] and TSC [15] have shown that the glass transition width is significantly broad in LCP's as compared to amorphous polymers and even high crystalline materials.

The TSDC spectra were analyzed using Bucci-FieschiGuidi analysis. Table 1 gives the relaxation parameters activation energy, relaxation strength, preexponential factor, charge released, and concentration of traps for $\beta$-relaxation, at various polarizing fields evaluated using BFG plots. Similarly, the values of various relaxation parameters for $\alpha$-relaxation are listed in Table 2. The relaxation parameters, activation energy, dielectric strength, and preexponential factor were calculated by taking the linear part of BFG plot. It is observed from Tables 1 and 2 that with the increase in polarizing field, activation energy decreases, while preexponential factor and relaxation strength increase. Also, the charge released and concentration of traps increase with the increase in polarizing field. The natural logarithm of preexponential factor is plotted as a function of activation energy for $\beta$ and $\alpha$-relaxations as shown in Figures 7 and 8, respectively. The linear variation between the $\ln \tau_{0}$ and activation energy indicates the presence of compensation effect for dipolar relaxations of thermotropic liquid crystal copolyester VectraA under present polarizing conditions, that is, at $T_{P}=80^{\circ} \mathrm{C}$ and for poling fields in the range of $3.8 \mathrm{kV} / \mathrm{cm}-19.2 \mathrm{kV} / \mathrm{cm}$. The compensation effect in thin films of Vectra-A and Vectra$\mathrm{E}$ was also reported by Collins and Long [2]. Compensation in terms of the linear dependence of $E$ and $\log \tau_{0}$ or in 


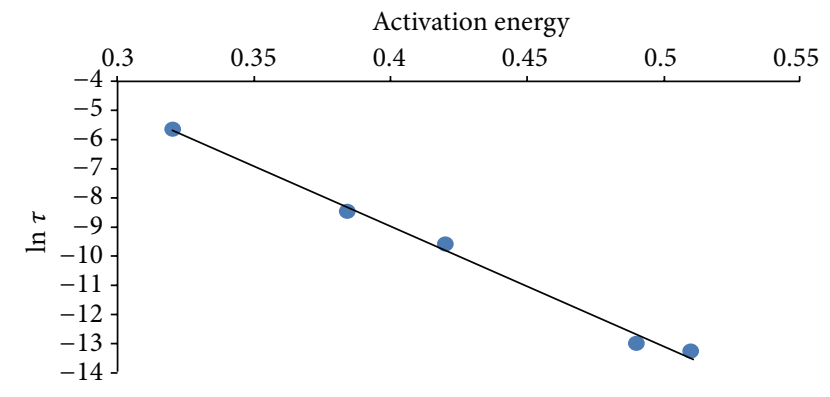

FIGURE 7: Variation of natural logarithm of preexponential factor with activation energy for $\beta$-relaxation.

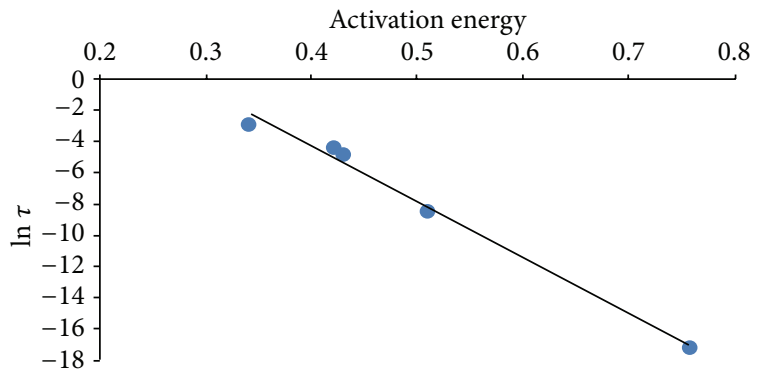

FIGURE 8: Variation of natural logarithm of preexponential factor with activation energy for $\alpha$-relaxation.

TABLE 2: Values of activation energy $(U)$, dipolar relaxation strength $\Delta \varepsilon$, preexponential factor $\left(\tau_{0}\right)$, charge released $(\mathrm{Q})$, and concentration of traps $\left(N_{t}\right)$ for $\alpha$-relaxation at poling temperature $T_{P}=80^{\circ} \mathrm{C}$ for various polarizing fields.

\begin{tabular}{lccccc}
\hline \multicolumn{5}{c}{$\alpha$-relaxation } \\
$\begin{array}{l}\text { Poling fields } \\
(\mathrm{kV} / \mathrm{cm})\end{array}$ & $U(\mathrm{eV})$ & $\Delta \varepsilon$ & $\tau_{0}(\mathrm{Sec})$ & $Q(\mathrm{C})$ & $N_{t}\left(\mathrm{~m}^{-3}\right)$ \\
\hline 3.8 & 0.76 & 2.8 & $3.75 \times 10^{-8}$ & $24 \times 10^{-10}$ & $6.53 \times 10^{12}$ \\
7.7 & 0.51 & 3.3 & $1.98 \times 10^{-4}$ & $58.80 \times 10^{-10}$ & $16 \times 10^{12}$ \\
11.5 & 0.43 & 4.6 & $7.48 \times 10^{-3}$ & $120 \times 10^{-10}$ & $16 \times 10^{12}$ \\
15.3 & 0.42 & 4.73 & $1.25 \times 10^{-2}$ & $162.87 \times 10^{-10}$ & $44.33 \times 10^{12}$ \\
19.2 & 0.34 & 4.99 & $5.60 \times 10^{-2}$ & $216.09 \times 10^{-10}$ & $58.82 \times 10^{12}$ \\
\hline
\end{tabular}

algebraically similar forms in terms of $\Delta H$ and $\Delta S$ is discussed in the literature $[17,18]$. The activated phenomena like solidstate diffusion in crystals and polymers, dielectric relaxation, conduction and thermally stimulated processes in polymers, and electronic conduction in amorphous semiconductors show the presence of compensation behavior $[19,20]$.

\section{Conclusions}

The TSDC investigations of liquid crystal copolyester VectraA reveals two dipolar relaxations, namely, $\beta-, \alpha$ - and a space charge $\delta$-relaxation in the temperature range from $15^{\circ} \mathrm{C}$ to $250^{\circ} \mathrm{C}$. The $\beta$-relaxation around $25^{\circ} \mathrm{C}$ is due to rotation of HNA groups, and the $\alpha$-relaxation around $110^{\circ} \mathrm{C}$ is associated with glass transition. The $\delta$ relaxation around $220^{\circ} \mathrm{C}$ is due to space charge trapping. The present studies indicate that positions and maximum currents of dipolar $\beta$ - and $\alpha$-relaxation are dependent on polarizing fields. The maximum current of $\beta$-, $\alpha$-, and $\delta$-relaxations increases with the increasing polarizing fields. There is also a shift in maximum current of $\beta$ - and $\alpha$-relaxations towards high temperature with the increase in polarizing field which is the characteristics of dipolar peaks. The linear variation between the activation energy and natural logarithm of preexponential factor indicates the presence of compensation effect for dipolar relaxations of thermotropic liquid crystal copolyester Vectra-A.

\section{References}

[1] J. V. Turnhout, "Electrets," in Topics in Applied Physics, G. M. Sessler, Ed., vol. 33, Springer, Berlin, Germany, 1980.

[2] G. Collins and B. Long, "Thermally stimulated current/relaxation map analysis of the relaxation processes in aromatic polyester, liquid crystal polymer film," Journal of Applied Polymer Science, vol. 53, no. 5, pp. 587-608, 1994.

[3] H. Shimizu, T. Kitano, and K. Nakayama, "Thermally stimulated depolarization current study on the glass transition of a liquid crystalline copolyester," Japanese Journal of Applied Physics, Part 2, vol. 35, no. 2, pp. L231-L233, 1996.

[4] B. Chowdhury, "Thermally stimulated processes in a liquid crystal polymer," Journal of Thermal Analysis and Calorimetry, vol. 56, no. 3, pp. 1167-1173, 1999.

[5] C. Bucci, R. Fieschi, and G. Guidi, "Ionic thermocurrents in dielectrics," Physical Review, vol. 148, no. 2, pp. 816-823, 1966.

[6] J. Vanderschueren and J. Gasiot, "Field-induced thermally stimulated currents," Thermally Stimulated Relaxations in Solids, vol. 37, pp. 135-223, 1979.

[7] M. S. Gaur, Ramlal, P. Shukla, P. Saxena, and R. K. Tiwari, “Thermally stimulated discharge current and fractional polarization studies in polyimide (Kapton-H) samples," Indian Journal of Pure and Applied Physics, vol. 46, no. 2, pp. 118-122, 2008.

[8] N. S. Yuksek, N. M. Gasanly, H. Ozkan, and O. Karci, “Trapping center parameters in $\mathrm{TlInS}_{2}$ layered crystals by thermally stimulated current measurements," Acta Physica Polonica A, vol. 106, no. 1, pp. 95-103, 2004.

[9] A. M. Donald and A. H. Windle, Liquid Crystalline Polymers, Cambridge University Press, 1992.

[10] X. J. Wang and Q. F. Zhou, Liquid Crystalline Polymers, World Scientific Publishing, 2004.

[11] D. S. Kalika and D. Y. Yoon, "Dielectric relaxation studies of poly(4-hydroxybenzoic acid) and copolyesters based on 4hydroxybenzoic acid and 6-hydroxy-2-naphthoic acid," Macromolecules, vol. 24, no. 11, pp. 3404-3412, 1991.

[12] S. J. Lukacs, “Temperature-dependent photophysical properties of a liquid-crystalline random copolyester," Journal of Physical Chemistry B, vol. 105, no. 17, pp. 3372-3377, 2001.

[13] R. W. Lenz, "Balancing mesogenic and non-mesogenic groups in the design of thermotropic polyesters," Faraday Discussions of the Chemical Society, vol. 79, pp. 21-32, 1985.

[14] H. Shimizu and K. Nakayama, "Thermally stimulated depolarization current study of molecular motions in polychlorotrifluoroethylene," Japanese Journal of Applied Physics, Part 1, vol. 28, no. 9, pp. 1616-1619, 1989.

[15] B. B. Sauer, R. Beckerbauer, and L. Wang, "Thermally stimulated current and DSC studies of the broadened glass transition in 
liquid crystalline polymers," Journal of Polymer Science B, vol. 31, no. 12, pp. 1861-1872, 1993.

[16] M. Y. Cao and B. Wunderlich, "Phase transitions in mesophase macromolecules. V. Transitions in poly(oxy-1,4-phenylene carbonyl-co-oxy-2,6-naphthaloyl)," Journal of Polymer Science $B$, vol. 23, pp. 521-535, 1985.

[17] B. B. Sauer and P. Avakian, "Cooperative relaxations in amorphous polymers studied by thermally stimulated current depolarization," Polymer, vol. 33, no. 24, pp. 5128-5142, 1992.

[18] J. P. Crine, "A new analysis of the results of thermally stimulated measurements in polymers," Journal of Applied Physics, vol. 66, no. 3, pp. 1308-1313, 1989.

[19] M. T. Ahmed and T. Fahmy, "Study of the relaxation phenomenon of Poly(vinyl chloride-co-vinylacetate-co-2hydroxypropyl acrylate)/Poly(methyl methacrylate) blends using TSDC-TS technique: dipole-dipole interaction approach," Journal of the Korean Physical Society, vol. 59, pp. 98-104, 2011.

[20] N. Mehta and A. Kumar, "Pre-exponential factor of Arrhenius equation for the isothermal crystallization of some Se-Ge, SeIn and Se-Te chalcogenide glasses," Journal of Materials Science, vol. 42, no. 2, pp. 490-494, 2007. 

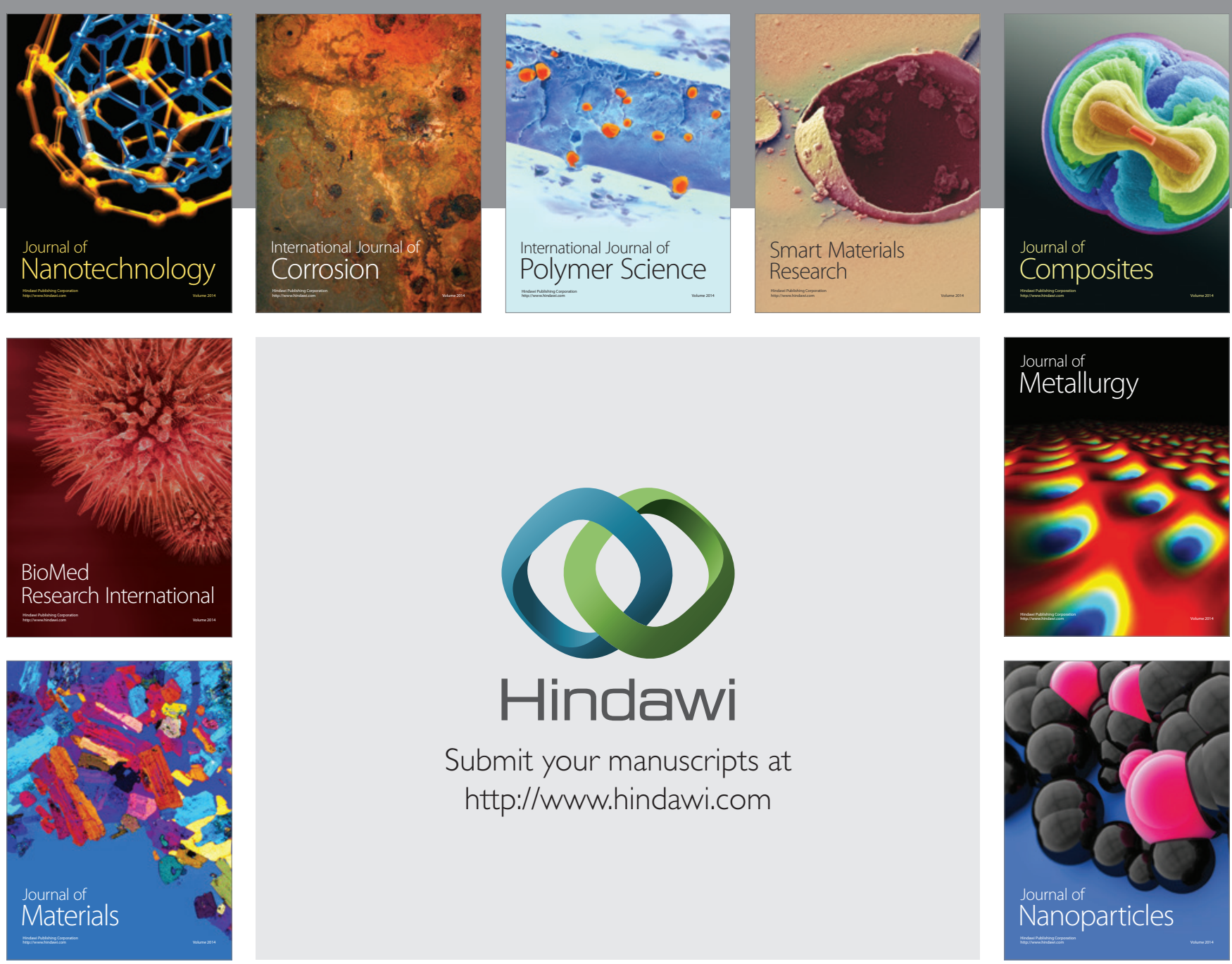

Submit your manuscripts at http://www.hindawi.com
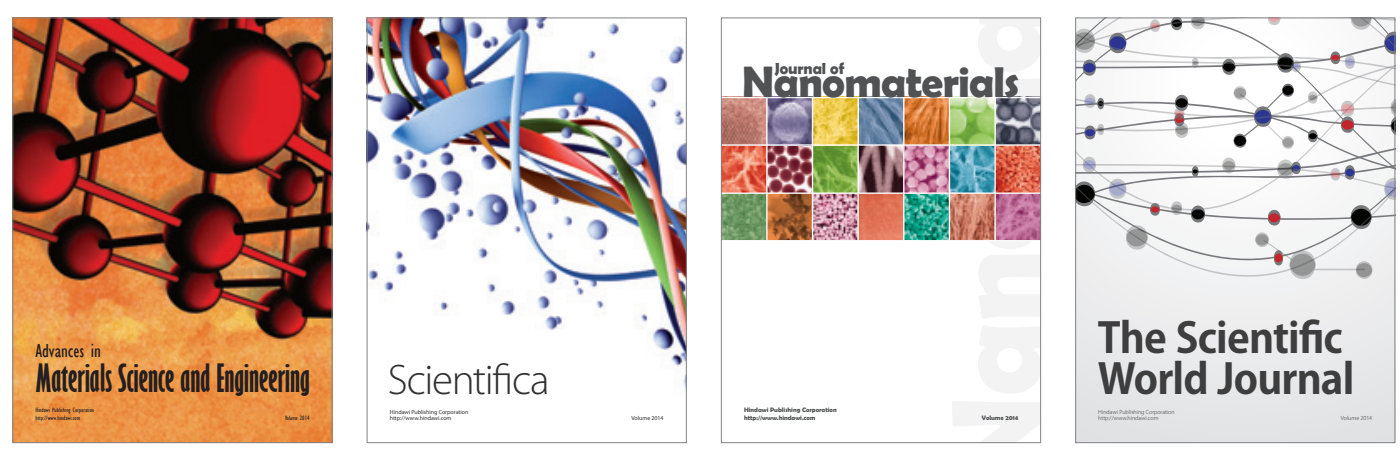

\section{The Scientific World Journal}
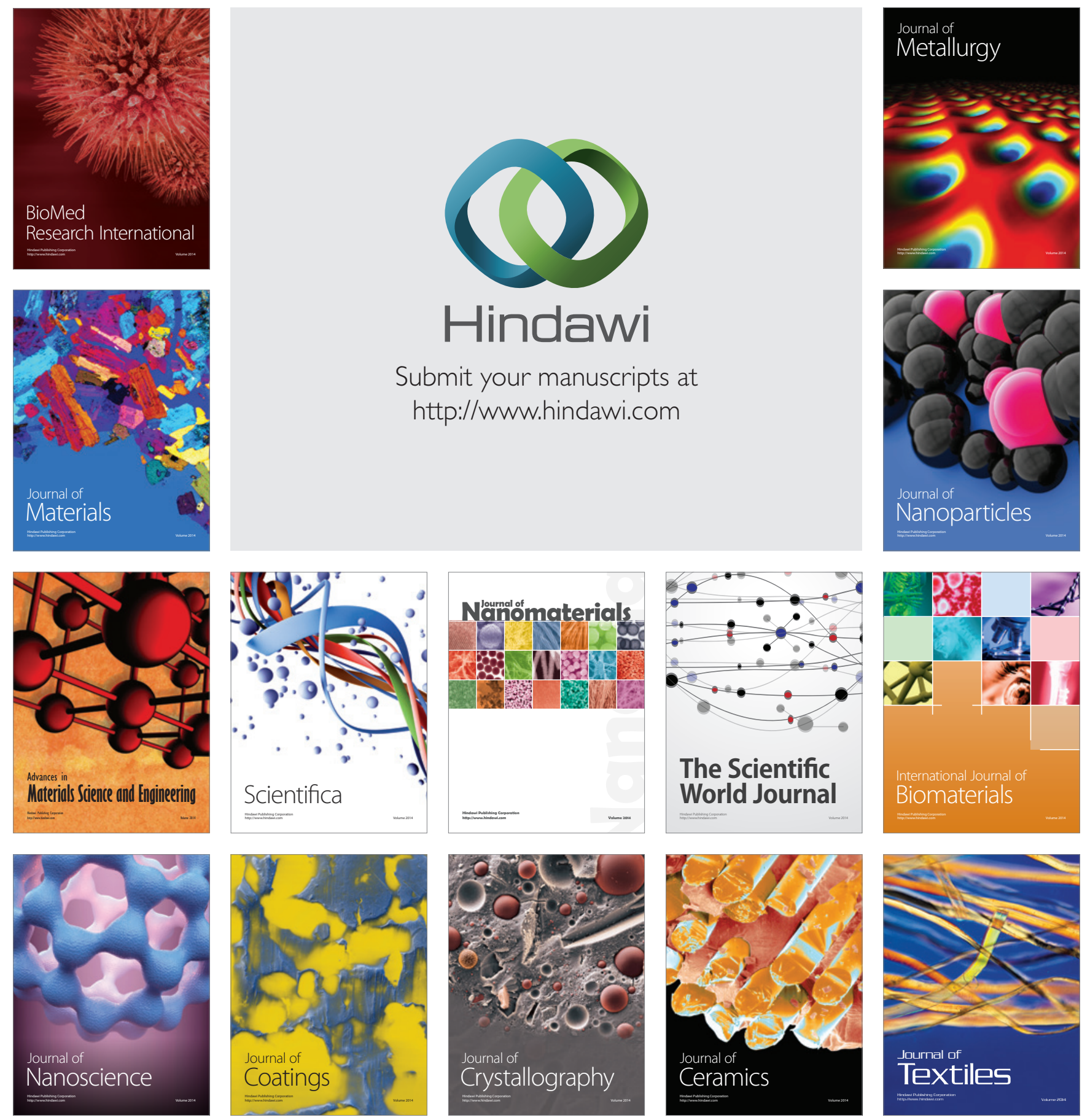